\title{
THE PHYSICS AND MATHEMATICAL ANALYSIS FOR THE TUT-T5 MONTE CARLO CODE
}

JANUARY 1960

CONTRACT AT-11-1-GEN-14

BETTIS ATOMIC POWER LABORATORY, PITTSBURGH, PA., OPERATED FOR THE U.S. ATOMIC ENERGY COMMISSION BY WESTINGHOUSE ELECTRIC CORPORATION

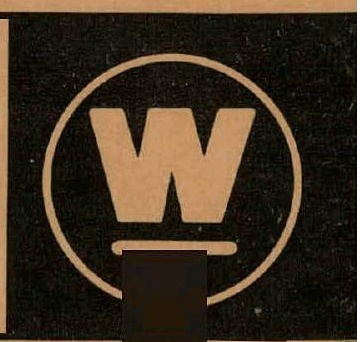




\section{DISCLAIMER}

This report was prepared as an account of work sponsored by an agency of the United States Government. Neither the United States Government nor any agency Thereof, nor any of their employees, makes any warranty, express or implied, or assumes any legal liability or responsibility for the accuracy, completeness, or usefulness of any information, apparatus, product, or process disclosed, or represents that its use would not infringe privately owned rights. Reference herein to any specific commercial product, process, or service by trade name, trademark, manufacturer, or otherwise does not necessarily constitute or imply its endorsement, recommendation, or favoring by the United States Government or any agency thereof. The views and opinions of authors expressed herein do not necessarily state or reflect those of the United States Government or any agency thereof. 


\section{DISCLAIMER}

Portions of this document may be illegible in electronic image products. Images are produced from the best available original document. 
UC-34: Physics and Mathematics

\title{
THE PHYSICS AND MATHEMATICAL ANALYSIS FOR THE TUT-T5 MONTE CARLO CODE
}

J. Spanier

January 1960

Contract AT-11-1-GEN-14

Price $\$ .50$

Available from the Office of Technical Services,

Department of Commerce,

Washington 25, D. C.

\section{NOTE}

This document is an interim memorandum prepared primarily for internal reference and does not represent a final expression of the opinion of Westinghouse. When this memorandum is distributed externally, it is with the express understanding that Westinghouse makes no representation as to completeness, accuracy, or usability of information contained therein.

\author{
BETTIS ATOMIC POWER LABORATORY \\ PITTSBURGH, PENNSYLVANIA \\ OPERATED FOR THE U. S. ATOMIC ENERGY COMMISSION BY \\ WESTINGHOUSE ELECTRIC CORPORATION
}




\section{SPECIAL EXTERNAL.DISTRIBUTION}

Manager, Pittsburgh Naval Reactors Operations Office, AEC

Argonne National Laboratory, W. F. Miller

Brookhaven National Laboratory, J. Chernick

Brookhaven National Laboratory, M. Rose

David Taylor Model Basin, H. Polachek

Knolls Atomic Power Laboratory, R. Ehrlich

Los Alamos Scientific Laboratory, B. Carlson

New Yurk University, R. Richtmyer

Oak Ridge National Laboratory, V. E. Anderson

Oak Ridge National Laboratory, A. Householder

University of California Kadiation Laboratory, L,ivermore, S. Fernbach

This report was prepared as an account of Government sponsored work. Neither the United States, nor the Commission, nor any person acting on behalf of the commission:

A. Makes any warranty or representation, expressed or implied, with respect to the accuracy, completeness, or usefulness of the information contained in this report, or that the use of any information, apparatus. method. or orocess dissinsed in this report may not infringe prlvately owned rights: or

B. Assumes any llablilities with respect to the use of, or for damages resulting from the use of any information, apparatus, method, or process disclosed in this report.

As used, in the above, "person acting on behalf of the commlssionn includes any employe or contractor of the commission, or employe of such contractor, to the extent that. such employe or contractor of the Commission, or employe of such contractor prepares, disseminates, or provides access to," any information pursuant to his "employment or contract with the Commission, or his employment with such contractor. 
CONTENTS

Page No.

I. INTRODUCTION

II. THE PHYSICAL PROCESS

3

III. THE INTEGRAL EQUATION: STATEMENT OF THE PROBLEM

IV. THE PROBABILITY MODEL

V. THE BASIC ESTIMATING VARIABLES 
The TUT-TS code for the 18M-704 estimates neutron capture probabilities in a one-energy, two-dimensional, rectangular cell model, using a Monte Carlo method. This report describes this problem and its solution in mathematical terms. The transport equation arising from the idealized physical model used in the code is derived and the problem is stated in terms of the solu. tion of this equation. The validity of the Monte Carlo solution is established by proving that the random variables involved are unbiased for processes with convergent Neumann series. These random variables sum the distances travelled by randomly selected particle histories and use these sums to estimate flux integrals over the various regions. Capture probabilities are then obtained through multiplication by the appropriate capture cross sections. The integrals which express the variances of these random variables appear too complicated to handle by analytic methods.

THE PHYSICS AND MATHEMATICAL ANALYSIS FOR THE TUT - T5 MONTE CARLO CODE

\section{J. Spanier}

\section{INTRODUCTION}

The TUT-T5 code for the IBM-704 (Ref 1) calculates, for a one-energy model, a regionwise distribution of neutron capture probabilities in a two-dimensional rectangular quarter-cell. The code is based on an analog Monte Carlo method in which random particle life histories are simulated using pseudo-random numbers, and the unknown capture probabilities are estimated by averages of weighting functions (random variables) associated with the histories. Although the specific technique used in TUT-T5, that of constructing estimates from the Monte Carlo track lengths, has been suggested before for use in similar problems (Ref 2, p 350), the author knows of no proof of the validity of the technique. * The method is sufficiently important to warrant a discussion in some detail. The present report concerns the mathematical analysis which forms the theoretical foundation for the TUT - T 5 code.

'Two major problems arise in connection with the analysis for any Monte Carlo calculation: (1) a proof that the calculation is unbiased and (2) an examination of the variance associated with the estimates of the unknown parameters. Stated loosely, the calculation is unbiased if the theoretical average of all the weights associated with all possible particle histories is exactly the quantity being estimated. Requiring that the calculation be unbiased imposes a somewhat arbitrary but certainly very plausible restriction on the class of admissible estimating random variables. The variance of the estimating random variable provides a measure of the deviation of the estimate, for a finite sample, from the unknown parameter. The variance thus provides a useful criterion for comparing the efficiencies of two competing methods of calculation. The estimate with the smaller variance will

*Recently, a proof has appeared in a preliminary draft: M. A. Martino and W. W. Stone, "TRAM, A Monte Carlo Thermal Neutron Code," KAPL-2039 (June 19, 1959). This proof is different from the present proof, since it is based on the introduction of a time-like variable and a general result on ergodic processes; moreover, the details of the proof have been omitted. The proof presented here is based on first principles. 
require fewer histories to achieve a fixed level of accuracy. In general, however, the smaller the variance, the more costly is the data processing per sample. Thus, these two effects must in practice be balanced to achieve maximum efficiency, i.e., minimum over-all computing time. In any event, a knowledge of the variance is always desirable but generally very difficult to obtain. In fact, for most problems it is as difficult to obtain sharp theoretical bounds on the variance as it is on the parameters being estimated. The digital calculation itself does provide an estimate of the variance, and this is then used in place of the variance to predict the expected error as a function of the size of the sample. The main objective of this report is to prove that the random variables used in TUT-T5 are unbiased; the integrals which express the variances appear too complex to handle in closed form.

An outline describing the development of the analysis for a general Monte Carlo neutron trans port code is presented in Ref 3, Section 6. According to this outline, the first step is to establish clearly the physical model being studied. The transition from physical model to mathematical model is then established via an integral equation, the integral form of the Boltzmann transport equation for the given process. Next, an abstract probability model is established in the form of a sample space of random walks $\Omega$, endowed with an appropriate probability measure $\mu$. The definition of $\Omega$ as a point set and the construction of a measure $\mu$ based on a random walk process, i.e., d melhud lor generating sample histories, are described in Ref 3, Section 7. Two types of measures $\mu$ are possible: the analog measure, in which the "events" undergone by each parlicle listory are exactly as likely in the probability, model as they are in the physical process, and non-analog measures, which distort the likelihoods of individual events in the probability model in an attempt to increase the efficiency of the calculation. The analog measure is uniquely determined by the transport equation for the physical model; however, a wide range of non-analog measures are possible in any given problem. Only the analog measure was considered in the formulation of 'TU'T-T'5, largely because the geometric complexity seemed to make non-analog techniques forbidding, if not impractical. Knowing the measure to use in setting up the sample space, it then remains to define for each parameter to be estimated, a random variable $\xi$ : that is, a real-valued, measurable function on $\Omega$. This function can be thought of as associating a "weight" $\xi(\mathrm{C})$ with every random walk chain $\mathrm{C}$ of $\Omega$. This weight is the C-estimate of the unknown parameter. It is assumed that each unknown parameter can be represented as an integral

$$
\bar{g}=\int_{\mathbf{R}} g(P) \psi(P) d P
$$

where $R$ is the physical phase space, $g(P)$ is a known function on $R_{i}$ and $\psi(P)$ is the transport theory collision density, the solution of the transport equation for the problem. Generating $\mathrm{N}$ random walk chains corresponds to selecting $N$ points, $C_{1}, \ldots, C_{N}$ of $\Omega$ using the constructed measure $\mu$. The average weight of these $\mathrm{N}$ chains $\bar{\xi}_{\mathrm{N}}$, defined by

$$
\bar{\xi}_{N}=\frac{1}{N} \sum_{i=1}^{N} \xi\left(C_{i}\right),
$$

converges by the Central Limit Theorem to the integral

$$
\bar{\xi}=\int_{\Omega} \xi \mathrm{d} \mu,
$$

where $d \mu$ is the differential of the probability measure $\mu$. It seems natural to require that $\bar{\xi}=\bar{g}$ and, if this is so, the random variable $\xi$ provides an unbiased estimate of the number $\bar{g}$. In this way, if the unbiased character of $\xi$ is established, the original integral $\bar{g}$ over phase space $R$ is replaced by the integral $\bar{\xi}$ over the sample space $\Omega$, and the latter integral is approximated by the quantities $\bar{\xi}_{\mathrm{N}}$ for sufficiently large $\mathrm{N}$. 
Observe that in this formulation the measure $\mu$ need not be analog to make the argument work. It is generally true that the function $\xi$ will have a simpler form when $\mu$ is analog measure than when $\mu$ is non-analog. In fact, for analog $\mu$ it may be very tempting to equate $\bar{g}$ and $\bar{\xi}$ through a physical argument. In this connection, it should be strongly emphasized that the sample space $\Omega$ and the measure $\mu$ have been constructed quite abstractly, and the fact that this probability model reproduces in some sense the physical model when $\mu$ is analog measure needs to be demonstrated rigorously. Thus, the statement, for the neutron $f l u x \phi(P)$ arising from a unit source, that

$$
\int_{R} \phi(P) d P=\bar{D}
$$

where $\bar{D}$ is the average total distance travelled from birth to death, is a statement concerning the behavior of a real neutron population in a certain physical system. On the other hand, the statement that

$$
\int_{\Omega} \xi \mathrm{d} \mu=\overline{\mathrm{d}}
$$

where $\xi$ is that random variable on $\Omega$ which assigns to each chain $C$ the total distance travelled be tween successive "collisions" and where $\overline{\mathbf{d}}$ is the expectation of $\xi$, is a statement about an abstract probability model. The equality of $\bar{d}$ and $\bar{D}$ is precisely the missing link which makes the transition from the one model to the other possible. Such problems arise in analyzing the TUT-T5 code, and it is hoped that the previous discussion serves to motivate the proof of the unbiased character of the random variables used in the code.

\section{THE PHYSICAL PROCESS}

In this section, the assumptions which govern the physical process studied in TUT-T5 are described. Thus, the geometry of the problem, the manner in which particles are introduced into this geometrical array, and the microscopic.laws which govern the behavior of the particles must be described. The integral equation which results from these assumptions is obtained in Section III.

The physical model under consideration gives rise to the one-energy integro-differential transport equation

$$
\begin{aligned}
& \nabla \cdot \underline{\omega} \phi(\underline{r}, \underline{\omega})+\Sigma_{t}(\underline{r}) \phi(\underline{r}, \underline{\omega})=\int_{\text {áll }}{ }_{d i r e c t i o n s} \underline{\omega}^{\prime} \phi\left(\underline{r}, \underline{\omega}^{\prime}\right)\left[\frac{\Sigma_{S}^{H}(\underline{r})}{4 \pi}\left(1+3 \bar{\mu}(\underline{r}) \underline{\omega} \cdot \underline{\omega}^{\prime}\right)\right. \\
& \left.+\frac{1}{4 \pi}\left(\Sigma_{s}(\underline{r})-\Sigma_{S}^{H}(\underline{r})\right)\right]+\frac{S(\underline{r})}{4 \pi},
\end{aligned}
$$

where

$\underline{r}$ is a spatial vector in three-dimensions,

$\underline{\omega} \quad$ Is a unit direction vector,

$\phi(\underline{r}, \underline{\omega})$ is the transport theory vector flux,

$\Sigma_{t}(\underline{r})$ is the total macroscopic cross section,

$\Sigma_{S}^{H}(\underline{r})$ is the macroscopic. srattering rross section for hydrogen,

$\Sigma_{S}(\underline{r})$ is the total macroscopic scattering cross section,

$\bar{\mu}(\underline{r}) \quad$ is the average cosine of the scattering angle (laboratory system) for hydrogen scattering,

$\underline{\omega} \cdot \underline{\omega}^{\prime}$ is the scalar product of the vectors $\underline{\omega}, \underline{\omega}$, and

$\mathrm{S}(\underline{\mathbf{r}})$ is the spatial source density, to be defined. 
Before defining the source density, it will be convenient to describe the geometry of the problem. In Ref 1 the geometry of TUT-T5 is described as two-dimensional with no variation in the $z$ direction. For the purposes of this report, a description in three dimensions will be given because the Monte Carlo code itself, being patterned after the physical model, treats all events as if they were taking place in three dimensions. As a result, the equations to be derived will be more pertinent if they contain all three spatial variables. Actually, the geometric configuration consists of an infinite lattice of rectangular cells in the $(x, y)$ plane, each cell assumed to be infinite in extent . in the $z$-direction. The basic cell will be defined in three dimensions by the following inequalities: $-\mathrm{M}_{1} \leq \mathrm{x} \leq \mathrm{M}_{1},-\mathrm{M}_{2} \leq \mathrm{y} \leq \mathrm{M}_{2},-1 / 2 \leq \mathrm{z} \leq 1 / 2$. The geometry of this cell is assumed to be symmetric about each of the planes $x=0, y=0$. These two planes divide the basic cell into four quarter-cells, the description of any one of which will thus serve to specify completely the entire lattice network. The basic quarter-cell is taken to be the one defined by the inequalities $0 \leq x \leq M_{1}, 0 \leq y \leq M_{2}$, $-1 / 2 \leq z \leq 1 / 2$. Because the geometry does not vary with $z$, the description of the basic quarter-cell will be complete when the two-dimensional intersection of the basic quarter-cell with the plane $z=0$ is described. This region of the $(x, y)$ plane may be subdivided into as many as 32 subrectangles, every subrectangle being considered homogeneous. Each subrectangle defines a region of the geometry. A generic region will be systematically denoted by the subscript $i, i=0, \ldots, N-1$.

Since it is not always desirable to estimate. capture probabilities for all regions, several regions may be grouped together to form a composition. The code automatically estimates capture probabilities for all compositions. A subscript $\mathrm{j}$ is used to denote a generic composition number, $j=0, \ldots, K-1 \leq N-1$.

Having described the geometric arrangement, it is possible to define the source density function $\mathrm{S}(\underline{\mathbf{r}})$ which occurs in Eq (2.1). This function is assumed to be constant in each region, the level in each region being arbitrary. Then,

$$
S(\underline{r}) \equiv S(x, y, z)=\frac{1}{\sum_{i=0}^{N-1} v_{i} S_{i}} \sum_{i=0}^{N-1} S_{i} x_{i}(x, y, z)
$$

is the defining equation for $S$ over the basic quarter-cell $0 \leq x \leq M_{1}, 0 \leq y \leq M_{2},-1 / 2 \leq z \leq 1 / 2$. In $\mathrm{Eq}(2.2), V_{i}$ is the volume of region $i, i=0, \ldots, N-1 ; x_{i}$ is the characteristic function of region i. defined by

$$
x_{i}(x, y, z)=\left\{\begin{array}{l}
1 \text { if }(x, y, z) \text { is in region } i \\
0 \text { otherwise; }
\end{array}\right.
$$

and $S_{i}$ is an arbitrary constant specifying the source level in region $i$. The function $S$ is then extended over all of three-dimensional space by symmetry and periodicity, as follows: and

$$
\left.\begin{array}{l}
S(x, y, z)=S(-x, y, z)=S(x,-y, z)=S(-x,-y, z), 0 \leq x \leq M_{1}, 0 \leq y \leq M_{2},-1 / 2 \leq z \leq 1 / 2 ; \\
S(x, y, z)=S\left(x \pm 2 M_{1}, y, z\right)=S\left(x, y \pm 2 M_{2}, z\right), \text { for all }(x, y, z) .
\end{array}\right\}
$$

Observe that the source term of $\mathrm{Eq} \mathrm{(2.1)} \mathrm{is} \mathrm{so} \mathrm{normalized} \mathrm{that} \mathrm{its} \mathrm{integral} \mathrm{over} \mathrm{all} \mathrm{regions} \mathrm{of} \mathrm{the}$ basic quarter-cell is unity.

The code considers only two general types of events upon collision of a neutron with a nucleus: elastic scattering and absorption. The relative probabilities for each event are specified by the macroscopic cross sections for the processes in question. Upon execution of a scattering collision, the distribution of new directions in the laboratory system is determined by the assumed distribution of scattering angles for the various elements. In TUT-T5, all elements except hydrogen are assumed 
to scatter isotropically in the laboratory system. As is evident from Eq (2. 1), the angular distribution arising from hydrogen scattering is assumed to have the form

$$
\Sigma_{s, i}^{H}(\mu)=\frac{\Sigma_{s, i}^{H}}{4 \pi}\left(1+3 \bar{\mu}_{i} \mu\right)
$$

in each region $i$, where $\mu$ is the cosine of the scattering angle in the laboratory system, and $\bar{\mu}_{i}$ is the average value of this cosine. Between collisions, the path lengths of particles measured in units of mean free path are distributed exponentially in the interval $0 \leq t \leq \infty$.

Since all particles are assumed to have the same energy throughout their lifetimes, energy considerations are irrelevant.

\section{THE INTEGRAL EQUATION: STATEMENT OF THE PROBLEM}

In this section the physical assumptions made in Section II are recast in the form of an integral equation-the integral form of the Boltzmann equation for the idealized process being studied. The capture probabilities estimated by the code are then expressed as definite integrals involving the solution of the Boltzmann equation. The problem solved by TUT-T5 is thereby stated mathematically.

Rather than obtain the integral equation via a transformation on the integro-differential equation (2. 1), a derivation from more general first principles will be outlined. The treatment used in this derivation is that of Ref 2, Appendix 2.

In the following, the general integral equation is discussed and then specialized to the case of TUT-T5.

Let $\underline{\mathbf{r}}$ denote a position vector in three-dimensional euclidean space, and let $\underline{E}$ denote a velocity vector, also in three-space. Writing $\underline{E}=\mathrm{E} \underline{\omega}$, then, $\underline{\omega}$ is a unit vector in the direction of motion and $E$ denotes the kinetic energy of the particle. The symbol $R$ represents the six-dimensional phase space of pairs $(\underline{r}, \underline{E})$.

The particle collision density, $\psi(\underline{r}, \underline{E})$, is defined so that the expected number of collisions of the particle with the medium contained in a volume $V$ of phase space is given by

$$
\iint_{V} \psi(\underline{r}, \underline{E}) d \underline{r} d \underline{F}
$$

where $d \underline{r} d \underline{E}$ is ordinary Lebesgue measure in $R$. The particle flux, $\phi(\underline{r}, \underline{E})$, is defined in terms of $\psi(\underline{r}, \underline{E})$ by the equation

$$
\psi(\underline{\mathbf{r}}, \underline{\mathrm{E}})=\Sigma_{\mathrm{t}}(\underline{\mathrm{r}}, \underline{\mathrm{E}}) \phi(\underline{\mathrm{r}}, \underline{\mathrm{E}}),
$$

where $\Sigma_{t}(\underline{r}, \underline{E})$ is the total macroocopic croos acction at $(\underline{\mathbf{r}}, \underline{E})$.

The general transport equation to be derived will have the form

$$
\psi(\underline{r}, \underline{E})=\iint_{R} \psi\left(\underline{r}^{\prime}, \underline{E}^{\prime}\right) K\left(\underline{r}^{\prime}, \underline{E}^{\prime} ; \underline{r}, \underline{E}\right) d \underline{E}^{\prime} \mathrm{d} \underline{r}^{\prime}+Q(\underline{r}, \underline{E}) .
$$

The source term, $Q(\underline{r}, \underline{E})$, represents the density of first collisions, and the integral term sums the contributions from particles which have already collided at least once.

The functions $Q$ and $K$ are now discussed in more detail.

The kernel $\mathrm{K}$ is most conveniently factored into a product of two functions, one dealing with changes in the spatial coordinates, the other dealing with changes in the velocity coordinates. Thus, the transport kernel $\mathrm{T}\left(\underline{r}^{\prime}, \underline{r} ; \underline{E}\right)$ is so defined that, for a particle leaving the source or a collision at $\left(\underline{r}^{\prime}, \underline{E}\right)$, the expected number of next collisions in the volume $V$ of position space is 


$$
\int_{V} T\left(\underline{r}^{\prime}, \underline{r} ; E\right) d \underline{r} \text {. }
$$

In similar fashion, the collision kernel $C\left(\underline{E^{\prime}}, \underline{E} ; \underline{r}\right)$ is so defined that, for a particle entering a collision at $\left(\underline{r}, \underline{E}^{\prime}\right)$, the expected number of particles leaving the collision within the volume $V$ of energy space is

$$
\int_{V} C\left(\underline{E}^{\prime}, \underline{E} ; r\right) d \underline{E} \text {. }
$$

By introducing an orthonormal set of vectors $\underline{\omega}$. $\underline{\omega}^{\prime}, \underline{\omega}^{\prime \prime}$ at $\underline{r}^{\prime}$, the function $T\left(\underline{\underline{r}} \underline{r}^{\prime}, \underline{E}\right.$ ) may be written

$$
\begin{array}{r}
T\left(\underline{r}^{\prime}, \underline{r} ; \underline{E}\right)=\Sigma_{t}(\underline{r}, \underline{E}) \exp \left[-\int_{0}^{\underline{\omega} \cdot\left(\underline{r}-\underline{r}^{\prime}\right)} \Sigma_{t}\left(\underline{r}^{\prime}+s \underline{\omega}, \underline{E}\right) d s\right] \delta\left(\underline{\omega} \cdot\left[\underline{r}-\underline{r}^{\prime}\right]\right) \\
\cdot \delta\left(\underline{\omega} \cdot\left[\underline{\underline{r}}-\underline{r}^{\prime}\right]\right) \eta\left(\underline{\omega} \cdot\left[\underline{r}-\underline{r}^{\prime}\right]\right) .
\end{array}
$$

where $\eta(x)=0$ if $x \leq 0, \eta(x)=1$ if $x>0$. The delta functions and the function $\eta$ guarantee that the vectors $\underline{r}$ lie along the forward trajectory of the path of the particle as it leaves $\underline{\underline{r}}$ with direction $\underline{\omega}$. The representation (3.3) results from the differential equation

$$
\nabla \cdot \frac{\underline{\omega} T\left(\underline{r}^{\prime} \cdot \underline{r} ; \underline{E}\right)}{\Sigma_{t}(\underline{r}, \underline{E})}+T\left(\underline{r}^{\prime}, \underline{r} ; \underline{E}\right)=\delta\left(\underline{r}^{\prime}-\underline{r}\right)
$$

satisfied by $T$, together with the condition that $T$ vanishes at infinity.

With this description of the function $T$, the source term $Q$ of Eq (3.2) may now be defined by

$$
Q(\underline{r}, \underline{E})=\int T\left(\underline{r}^{\prime}, \underline{r} ; \underline{E}\right) S\left(\underline{r}^{\prime}, \underline{E}\right) d \underline{r}^{\prime} \text {. }
$$

the integral being taken over all of position space and the function $S\left(\underline{r^{\prime}}, \underline{E}\right)$ being the physical source density of particles at $\left(\underline{r}^{\prime}, \underline{E}\right)$. The function $Q(\underline{r}, \underline{E})$ now may be seen to represent the rate of collision at $(\underline{r}, \underline{E})$ of particles which have been injected into the system at $\left(\underline{r}^{\prime}, \underline{E}\right)$ and then transported to $(\underline{r}, \underline{E})$ by the transport kernel ' 1 '.

The collision kernel $\mathrm{C}$ is more difficult to describe in general than the kernel $\mathrm{T}$ because, on a given collision, many types of events are competing with various probabilities. It is usual to express $\mathrm{C}$ as

$$
C^{\prime}=\Sigma p_{i} C_{i}
$$

where $p_{i}$ is the probability of an event of type $i$, and $C_{i}$ is a corresponding kernel for that event. Explicit forms for the $C_{i}$ must be obtained from the laws of collision mechanics in each separate case.

The kernel $K\left(\underline{r}^{\prime}, \underline{E} ; \underline{r}, \underline{E}\right)$ of Eq (3.2) is now defined by

$$
K\left(\underline{r}^{\prime}, \underline{E^{\prime}} ; \underline{r}, \underline{E}\right)=C\left(\underline{E}^{\prime}, \underline{E} ; \underline{\underline{r}}^{\prime}\right) l^{\prime}\left(\underline{r}^{\prime}, \underline{r} ; \underline{\underline{b}}\right) \text {. }
$$

so that $K\left(\underline{r}^{\prime}, \underline{E} \underline{E}^{\prime}, \underline{r}, \underline{E}\right)$ transports particles from the state $\left(\underline{r}^{\prime}, \underline{E}^{\prime}\right)$ to $(\underline{r}, \underline{E})$ via a collision at the space point $\underline{r}^{\prime}$, followed by transmission from $\underline{\underline{r}}^{\prime}$ to $r$ at the velocity vector $\underline{E}$. An integral equation similar to (3.2) is satisfied by the flux, $\phi(\underline{\underline{r}}, \underline{E})$; however, this report will deal exclusively with the equation for the collision density.

In the problem considered by the code, since there is no energy dependence, the collision density $\psi$ is a function of five variables. To denote a unit vector in the direction of flight of the particle, $\underline{\omega}$ is again used and polar coordinates are used to describe the components of this vector. Thus, $\underline{\omega}=(\theta, \phi)$ specifies that $\theta$ is the polar angle of $\underline{\omega}, 0 \leq \theta \leq \pi$, measured from the positive $z$-axis in euclidean three-space, and $\phi$ is the angle between the positive $x$-axis and the projection of $\underline{\omega}$ on the $(x, y)$ plane, $0 \leq \phi \leq 2 \pi$. For spatial components, rectangular coordinates in three-space will be used and written $\underline{r}=(x, y, z)$. 
The physical source density for TUT-T5 has already been defined in Section II; it is the function

$$
S(\underline{r}, \underline{\omega})=\frac{S(\underline{r})}{4 \pi}
$$

where $S(\underline{r})$ is the function defined by Eqs (2.2) and (2.3). The transport kernel $\mathrm{T}$ has been defined in general in Eq (3.3); thus, it remains only to define the collision kernel $\mathrm{C}$ to.specify the integral equation for the code. In view of the assumptions made in Section II concerning the scattering laws of the various elements, $\mathrm{C}$ is given by

$$
\mathrm{C}\left(\underline{\omega}^{\prime}, \underline{\omega} ; \underline{r}\right)=\frac{\Sigma_{S}(\underline{r})}{\Sigma_{t}(\underline{r})}\left[\frac{\Sigma_{s}^{H}(\underline{r})}{\Sigma_{S}(\underline{r})}\left\{\frac{1+3 \bar{\mu}(\underline{r}) \underline{\omega}=\underline{\omega}^{\prime}}{4 \pi}\right\}+\frac{\Sigma_{s}(\underline{r})-\Sigma_{s}^{H}(\underline{r})}{4 \pi \Sigma_{s}(\underline{r})}\right],
$$

where $\Sigma_{s}(\underline{r}), \Sigma_{t}(\underline{r}), \Sigma_{s}^{H}(\underline{r}), \bar{\mu}(\underline{r})$ have the same meaning as in Section II. The full integral equation satisfied by the collision density in TUT-T5 is, therefore, the equation

$$
\begin{aligned}
& \psi(\underline{r}, \underline{\omega})=\iint_{R} \psi\left(\underline{r}^{\prime}, \underline{\omega}^{\prime}\right) \frac{\Sigma_{t}(\underline{r})}{\Sigma_{t}\left(\underline{r}^{\prime}\right)}\left[\frac{\Sigma_{s}^{H}\left(\underline{r}^{\prime}\right)}{4 \pi}\left\{1+3 \bar{\mu}\left(\underline{r}^{\prime}\right) \underline{\omega} \cdot \underline{\omega}^{\prime}\right\}+\frac{\Sigma_{s}\left(\underline{r}^{\prime}\right)-\Sigma_{s}^{H}\left(\underline{r}^{\prime}\right)}{4 \pi}\right] \\
& \cdot \exp \left[-\int_{0}^{\underline{\omega} \cdot \underline{\left(\underline{r}-\underline{r}^{\prime}\right)}} \Sigma_{t}\left(\underline{r}^{\prime}+s \underline{\omega}\right) d s\right] \delta\left(\underline{\omega}^{\prime} \cdot\left[\underline{r}-\underline{r}^{\prime}\right]\right) \delta\left(\underline{\omega}^{\prime \prime} \cdot\left[\underline{r}-\underline{r}^{\prime}\right]\right) \eta\left(\underline{\omega} \cdot\left[\underline{r}-\underline{r}^{\prime}\right]\right) d \underline{\omega}^{\prime} d \underline{r}^{\prime} \\
& +\int \Sigma_{t}(\underline{r}) \exp \left[-\int_{0}^{\underline{\omega} \cdot\left(\underline{r}-\underline{r}^{\prime}\right)} \Sigma_{t}\left(\underline{r}^{\prime}+s \underline{\omega}\right) d s\right] \delta\left(\underline{\omega}^{\prime} \cdot\left[\underline{r}-\underline{r}^{\prime}\right]\right) \delta\left(\underline{\omega}^{\prime \prime} \cdot\left[\underline{r}-\underline{r}^{\prime}\right]\right) \eta\left(\underline{\omega} \cdot\left[\underline{r}-\underline{r}^{\prime}\right]\right) \frac{S\left(\underline{r}^{\prime}\right)}{4 \pi} \cdot d \underline{r}^{\prime} \cdot
\end{aligned}
$$

where $S(\underline{r})$ is the source density defined by Eqs (2, 2) and (2,3).

The physical assumptions guarantee that the solution $\psi(\underline{r}, \underline{\omega})=\psi(x, y, z, \theta, \phi)$ will be a periodic function of the variables $x$ and $y$ and will be independent of $z$. Interest will be focused on the solution in the range of values $0 \leq x \leq M_{1}, 0 \leq y \leq M_{2},-1 / 2 \leq z \leq 1 / 2,0 \leq \theta \leq \pi$, and $0 \leq \phi \leq 2 \pi$.

The problem solved by the code is the estimation of each of the integrals

$$
p_{j}=\iint_{R} x_{j}(\underline{r}) \frac{\Sigma_{a}(\underline{r})}{\Sigma_{l}(\underline{r})} \psi(\underline{r}, \underline{\omega}) \mathrm{d} \underline{r} \mathrm{~d} \underline{\omega} ; j=0, \ldots, K-1,
$$

where $x_{j}(\underline{r})$ is the characteristic function for composition $j$ of the basic quarter-cell, and $\psi(\underline{r}, \underline{\omega})$ satisfies $\mathrm{Eq}(3.8)$. The number $\mathrm{p}_{\mathrm{j}}$ shall be referred to as the capture probability for composition $j$.

In the analysis given later, it will be necessary that the source term appearing on the right side of Eq (3.8) integrate to unity over all of phase. space. The integral in question is given by

$$
\iiint_{R} T\left(\underline{r}^{\prime}, \underline{r} ; \underline{\omega}\right) \frac{S\left(\underline{r}^{\prime}\right)}{4 \pi} d \underline{r}^{\prime} d \underline{r} \mathrm{~d} \underline{\omega}
$$

the $\underline{r}^{\prime}$ integral being over the basic quarter-cell. From the definition of $\mathrm{T}$ through the differential equation (3.4), it is easy to show that

$$
\int_{\text {all space }} T\left(\underline{r}^{\prime}, \underline{r} ; \underline{\omega}\right) d \underline{r}=1 \text {. }
$$

Then, inverting the order of integration in (3.10),

$$
\iiint_{R} T\left(\underline{r}^{\prime}, \underline{r} ; \underline{\omega}\right) \frac{S\left(\underline{r}^{\prime}\right)}{4 \pi} d \underline{r}^{\prime} d \underline{r} d \underline{d}=\iint_{\text {quarter - cell }} \frac{S\left(\underline{r}^{\prime}\right)}{4 \pi} \mathrm{d} \underline{r}^{\prime} \mathrm{d} \underline{\omega}=1 .
$$


Equation (3.11) states that the total birth rate has been normalized to unity over the 'basic quartercell. Since the source is periodic, its integral over all of phase space is unbounded. To avoid an unbounded source, the present formulation is replaced with a mathematically equivalent one which has the proper normalizations. This is done by placing the required source in Eq (3.8) and altering the definition of the numbers $p_{j}$.

Consider a function $S_{N}(\underline{r})$ which coincides with $S(\underline{r})$ over the basic quarter-cell and which vanishes everywhere else. Let $\psi_{N}(\underline{r}, \underline{\omega})$ be the function which satisfies Eq (3.8) when $S\left(\underline{r}^{\prime}\right)$ is replaced by $S_{N}\left(\underline{r}^{\prime}\right)$ on the right side. The collision density $\psi_{N}(\underline{r}, \underline{\omega})$ is no longer a periodic function on phase space. Indeed, $\psi_{N}$ vanishes at infinity because the source $S_{N}$ is now confined to the basic quartercell. It is clear from the symmetry of the geometry that the numbers $p_{j}$ may be defined in terms of the new function $\psi_{\mathrm{N}}$ by the equations

$$
p_{j}=\iint_{R} x_{j}^{*}(\underline{r}) \frac{\Sigma_{a}(\underline{r})}{\sum_{t}(\underline{r})} \psi_{N}(\underline{r}, \underline{\omega}) d \underline{r} d \underline{\omega}, j=0, \ldots, K-1,
$$

where $x_{j}^{*}(\underline{r})$ is now the characteristic function of the union of all the replicas of composition $j$ over all the quarter-cells of the lattice network. Integrals of this type will be dealt with in the remainder of this report.

The results of this section may be summarized in the following way: initially, the problem is stated in terms of a source which is periodic, taking on the same values in each replica of the basic quarter-cell. The collision density arising from such a source is also periodic, and the capture probabilities may be defined as weighted integrals of the collision density, restricted to a single basic quarter-cell. The source in every quarter-cell contributes to these weighted integrals, by amounts which diminish as the distance from the quarter-cell to the basic quarter-cell increases. Now, if the source is confined to the basic quarter-cell, the source will make contributions to the collision density over all phase space in such a way that, if the weighted integrals are summed over all of phase space, the original capture probabilities will result. Since later analysis requires a unit source, this point of view is adopted in formulating the problem solved by the code. The code thus estimates the integrals $(3.12)$, where $\psi_{N}(\underline{r}, \underline{\omega})$ satisfies

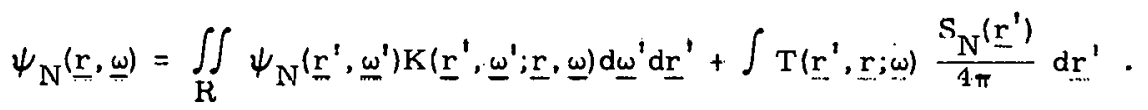

The function $\mathrm{K}\left(\underline{r}^{\prime}, \underline{\omega}^{\prime} ; \underline{r}, \omega\right)$ is the kernel of Eq (3.8) and the source terms are identical except for the. replacement of $S\left(\underline{r}^{\prime}\right)$ by $S_{N}\left(\underline{r}^{\prime}\right)$. The numbers $p_{j}$ defined by Eq (3.12) coincide with those defined by Eq (3.9).

Finally, in vlew of the balance between births and captures in the steady-state (in the absence of leakage),

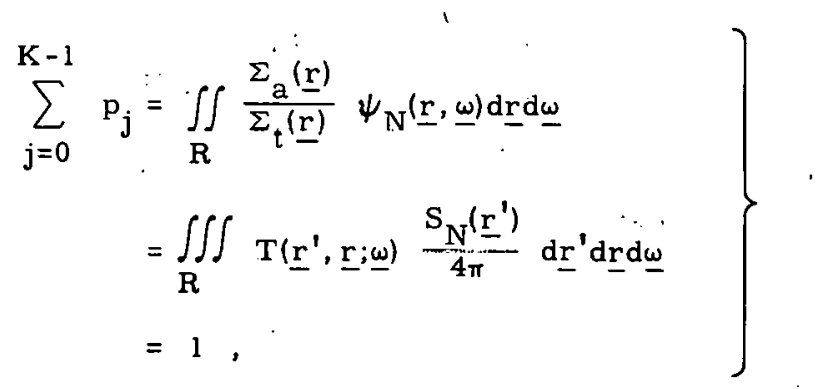

which shows that the numbers $p_{j}$ may truly be regarded as probabilities of capture in composition $j$. IV. THE PROBABILITY MODEL

In this section the probability model used in the formulation of the code is defined. As indicated in the introduction, a sample space $\Omega$ of random walk chains, together with a measure on the space $\Omega$ 
must be defined. In effect, the measure prescribes a method of generating sequences of collision. points, each sequence specifying a random walk made by a Monte Carlo particle history. How each history obtained in this way is to be weighted for the estimation of the numbers $p_{j}$ defined by Eq (3. 12) is shown in Section $\mathrm{V}$ where the random variables are defined.

As mentioned in Section I of this report, only the analog model was considered in the formulation of TUT-T5. This means that the particle histories generated by the code will simulate as closely as possible the life histories of randomly selected real particles, as they might occur in the idealized physical model treated here. The method for defining two sequences of functions, $f_{n}$; $p_{n}, n=0$, $1, \ldots$, from which the analog measure $\mu$ may be constructed, was given in Ref 3 . As expected, these functions are uniquely determined by the source and kernel of Eq (3.13). Intuitively, the function $f_{n}$ is a probability density function on $R^{n+l}$, the set of all $(n+1)$-tuples of points of phase space $R$, which gives the probability density of the sequence $\left(\underline{r}_{0}, \underline{\omega}_{0} ; \underline{r}_{1}, \underline{\omega}_{1} ; \ldots ; \underline{r}_{n}, \underline{\omega}_{n}\right)$ of collision points. The function $p_{n}$ is a non-negative function on $\mathbf{R}$ giving the probability of terminating the history at any given point of phase space. For the present analog process, these functions are defined as follows:

$$
\begin{aligned}
& \mathrm{f}_{0}\left(\underline{r}_{0}, \underline{\omega}_{0}\right)=\int \mathrm{T}\left(\underline{r}^{\prime}, \underline{r}_{0} ; \underline{\omega}_{0}\right) \frac{\mathrm{S}_{\mathrm{N}}\left(\underline{\underline{r}}^{\prime}\right)}{4 \pi} \mathrm{d} \underline{r}^{\prime} \text {, } \\
& \left.\begin{array}{rl}
\mathrm{f}_{\mathrm{n}}\left(\underline{\mathrm{r}}_{0}, \underline{\omega}_{0} ; \ldots ; \underline{\mathrm{r}}_{\mathrm{n}}, \underline{\omega}_{\mathrm{n}}\right) & =\left[\prod_{\ell=1}^{\mathrm{n}} \frac{\mathrm{K}\left(\underline{\mathrm{r}}_{\ell-1}, \underline{\omega}_{\ell-1} ; \underline{\mathrm{r}}_{\ell}, \underline{\omega}_{\ell}\right)}{\iint_{\mathrm{K}} \mathrm{K}\left(\underline{\mathrm{r}}_{\ell-1}, \underline{\underline{\omega}}_{\ell-1} ; \underline{\mathrm{r}}_{\ell}, \underline{\omega}_{\ell}\right) \mathrm{d} \underline{\mathrm{r}}_{\ell} \mathrm{d} \underline{\omega}_{\ell}}\right] \mathrm{f}_{0}\left(\underline{\mathrm{r}}_{0}, \underline{\omega}_{0}\right) \\
& =\left[\prod_{\ell=1}^{\mathrm{n}} \frac{\mathrm{K}\left(\underline{\mathrm{r}}_{\ell-1}, \underline{\omega}_{\ell-1} ; \underline{\mathrm{r}}_{\ell}, \underline{\omega}_{\ell}\right)}{1-\mathrm{p}_{\ell-1}\left(\underline{\mathrm{r}}_{\ell-1}, \underline{\omega}_{\ell-1}\right)}\right] \mathrm{f}_{0}\left(\underline{\mathrm{r}}_{0}, \underline{\omega}_{0}\right),
\end{array}\right\}
\end{aligned}
$$

where

$$
p_{\ell}\left(\underline{r}_{\ell} \underline{\omega}_{\ell}\right)=1-\frac{\Sigma_{s}\left(\underline{r}_{\ell}\right)}{\Sigma_{t}\left(\underline{r}_{\ell}\right)}=\frac{\Sigma_{a}\left(\underline{r}_{\ell}\right)}{\Sigma_{t}\left(\underline{r}_{\ell}\right)}
$$

and where $\mathrm{K}$ is the kernel of $\mathrm{Eq}_{\mathrm{q}}(3.13)$. Observe that the relation $(4.3)$ implies the equality

$$
\iint_{\mathrm{R}} \mathrm{K}\left(\underline{r}_{\ell-1}, \underline{\omega}_{\ell-1} ; \mathrm{r}_{\ell}, \underline{\omega}_{\ell}\right) \mathrm{d} \underline{r}_{\ell} \mathrm{d} \underline{\omega}_{\ell}=\frac{\Sigma{ }_{\mathrm{S}}\left(\underline{r}_{\ell-1}\right)}{\Sigma_{t}\left(\underline{r}_{\ell-1}\right)},
$$

which follows from the detailed definition of the kernel $\mathrm{K}$ given in Section 3 .

The function $f_{0}$ is the normalized density of first collisions; that is, the source term of Eq (3. 13) evaluated at $\left(\underline{r}_{0}, \underline{\omega}_{0}\right)$. The function $\mathrm{f}_{\mathrm{n}}$ is clearly properly normalized over $\mathrm{R}^{\mathrm{n}+1}$.

The functions $f_{n}, p_{n}$ are used to generate sample histories in the following way: first, an initial state $\left(\underline{r}_{0}, \underline{\omega}_{0}\right)$ is selected from the density function $\mathrm{f}_{0}\left(\underline{r}_{0}, \underline{\omega}_{0}\right)$ according to well-known sampling methods, choosing one real coordinate at a time. The state $\left(\underline{r}_{0}, \underline{\omega}_{0}\right)$ is the point of the first collision of the initial history. This history will terminate at $\left(\underline{r}_{0}, \underline{\omega}_{0}\right)$, with probability $\dot{\mu}_{0}\left(\underline{r}_{0}, \underline{\omega}_{0}\right)=$ $\Sigma_{a}\left(\underline{r}_{0}\right) / \Sigma_{t}\left(\underline{r}_{0}\right)$ and will continue beyond that state with probability $\Sigma_{s}\left(\underline{r}_{0}\right) / \Sigma_{t}\left(\underline{r}_{0}\right)$. A random choice is made to determine this decision. If the history does not terminate at $\left(\underline{r}_{0}, \underline{\omega}_{0}\right)$, the next collision point $\left(\underline{r}_{1}, \underline{\omega}_{1}\right)$ is chosen from the conditional probability density function

$$
\frac{\underline{f}_{1}\left(\underline{r}_{0}, \underline{\omega}_{0} ; \underline{r}_{1}, \underline{\omega}_{1}\right)}{f_{0}\left(\underline{r}_{0}, \underline{\omega}_{0}\right)}=\frac{K\left(\underline{\underline{w}}_{0}, \underline{w}_{0} ; \underline{\underline{r}}_{1}, \underline{\omega}_{1}\right)}{\frac{\sum_{s}\left(\underline{r}_{0}\right)}{\Sigma}},
$$

*See Ref 3: Eqs (8.3), (8.4), and (8.5). 
where $\left(\underline{\mathbf{r}}_{0}, \underline{\omega}_{0}\right)$ is the previously determined initial collision point. In general, if the sequence $\underline{r}_{0}$. $\underline{\omega}_{0}, \ldots, \underline{r}_{n-1}, \underline{\omega}_{n-1}$ of collision points has been specified and if it is decided by a random choice not to terminate the history at $\underline{\underline{r}}_{n-1}, \underline{\omega}_{n-1}$, the next chain point is selected from the conditional density function

$$
\frac{f_{n}\left(\underline{r}_{0}, \underline{\omega}_{0} ; \ldots ; \underline{r}_{n}, \underline{\omega}_{n}\right)}{f_{n-1}\left(\underline{r}_{0} \cdot \underline{\omega}_{0} ; \ldots ; \underline{r}_{n-1}, \underline{\omega}_{n-1}\right)}=\frac{K\left(\underline{r}_{n-1}, \underline{\omega}_{n-1} ; \underline{r}_{n}, \underline{\omega}_{n}\right)}{\frac{\Sigma s\left(\underline{r}_{n-1}\right)}{\Sigma}} \text {. }
$$

Each history is followed until capture ultimately occurs. This process is repeated until a large number of histories have been generated.

The space $\Omega$ of random walk chains is defined as the infinite product space

$$
\Omega=\prod_{i=0}^{\infty} x_{i}, \text { where } x_{i}=R \times\{P, Q\}, i=0,1,2, \ldots
$$

and where $K$ is the phase space and $\{P, Q\}$ denotes a two point space. A point of $\Omega$ is then an infinite sequence $\left\{\left(\alpha_{n}, \alpha_{1}, \ldots\right)\right\}$, where each $\alpha_{i}$ specifies a state $\left(\underline{r}_{i}, \underline{\omega}_{i}\right)$ of $R$, chosen as described in the preceding paragraph, together with a "distinguished" state, $P$ or $Q$. The designation of the state $P$ means that the chain is regarded as terminated at that collision, while the state $Q$ corresponds to non-termination. A particle which is terminated in a given state after $n$ collisions remains in that state for all further collisions with probability one.

The analog measure $\mu$ on $\Omega$ was defined in Ref 3 , Section 7 , and is based on the sequences $f_{n}$, $p_{n}$ of Eqs (4.1), (4.2), and (4.3) of the present report. More generally, the method for constructing a probability measure on $\Omega$ based on more general considerations has been given, the analog measure resulting as a special case of this construction. In any event, this construction will not be repeated here and the existence of the analog measure $\mu$ will henceforth be assumed.

\section{THE B $\Lambda$ SIC ESTIMATING VARIABLES}

In this section the random variables used in TUT-T5 are defined and; modulo certain convergence assumptions, it is. shown that they are unbiased.

If, as in Section IV, the density of first collisions is denoted by $f_{0}(\underline{r}, \underline{\omega})$, $i . e$. , the source term of Eq (3.13), then that equation becomes

$$
\psi_{N}(\underline{r}, \underline{\omega})=\iint_{R} \psi_{N}\left(\underline{r}^{\prime}, \underline{\omega}^{\prime}\right) K\left(\underline{r}^{\prime}, \underline{\omega}^{\prime} ; \underline{r}, \underline{\omega}\right) d \underline{r}^{\prime} \mathrm{d} \underline{\omega}^{\prime}+f_{0}(\underline{r}, \underline{\omega}) .
$$

The function

$$
g_{j}(\underline{r}, \underline{w})=x_{j}^{*}(\underline{r}) \frac{\Sigma_{a}(\underline{r})}{\Sigma_{t}(\underline{r})}, j=0, \ldots, k-1,
$$

is defined so that, from Eq (3.12),

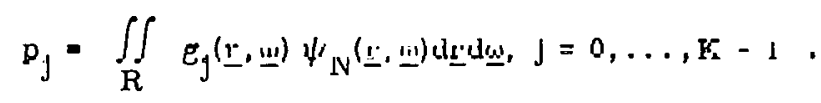

For each $\mathrm{j}=0, \ldots \mathrm{k}-1$, an adjoint integral equation is defined by

$$
\eta_{j}(\underline{r}, \underline{\omega})=\iint_{R} \eta_{j}\left(\underline{r}^{\prime}, \underline{\omega}^{\prime}\right) K\left(\underline{r}, \underline{\omega} ; \underline{r}^{\prime}, \underline{\omega}^{\prime}\right) d \underline{r}^{\prime} d \underline{\omega}^{\prime}+g_{j}(\underline{r}, \underline{\omega}) .
$$

Notice that in Eq (5.4), the arguments have been transposed in the kernel and the source term has been replaced by $g_{j}$. Equation (5.4) is evidently an integral equation of the same type as (5.1). The solution, $\eta_{j}(\underline{r}, \underline{\omega})$, may be interpreted as the expected contribution of a particle at $(\underline{\mathbf{r}}, \underline{\omega})$ to the capture 
probability $p_{j}$. This expected contribution is composed of a direct contribution (no further collisions), the source term of (5.4), plus an indirect contribution-the integral term involving the transposed kernel.

The reasons for introducing the adjoint equation are somewhat technical. First, the result used to prove that the TUT-T5 estimators are unbiased involves an assumption concerning the Neumann series of the adjoint equation. Second, the technique involved-namely, that of using Monte Carlo track lengths to estimate the numbers $p_{j}$, actually is a special case of a more general device to obtain Monte Carlo estimates of the solution $\eta_{j}$ of the adjoint equation. This theory has been developed in detail in Ref 3, Section 8. In the present report, the general result is used without giving the background or any details.

The assumption is now made that the Neumann series

$$
\mathrm{f}_{0}(\underline{\mathrm{r}}, \underline{\omega})+\sum_{\mathrm{k}=1}^{\infty} \mathrm{I}_{\mathrm{k}}(\underline{\mathrm{r}}, \underline{\omega})
$$

converges to $\psi_{N}(\underline{r}, \underline{\omega})$ for all $(\underline{\mathbf{r}}, \underline{\omega})$ of $R$, where

$$
\begin{array}{r}
I_{k}(\underline{r}, \underline{\omega})=\int_{R} \cdots \int_{R} K\left(\underline{r}_{k-1}, \underline{\omega}_{k-1} ; \underline{\underline{r}}, \underline{\omega}\right) K\left(\underline{r}_{k-2}, \underline{\omega}_{k-2} ; \underline{r}_{k-1}, \underline{\omega}_{k-1}\right) \cdots \mathrm{K}\left(\underline{r}_{0}, \underline{\omega}_{0} ; \underline{r}_{1}, \underline{\omega}_{1}\right) \\
\quad \mathrm{f}_{0}\left(\underline{r}_{0}, \underline{\omega}_{0}\right) d \underline{r}_{0} \cdots \mathrm{d} \underline{r}_{k-1} d \underline{\omega}_{0} \cdots d \underline{\omega}_{k-1} .
\end{array}
$$

The assumption is also made that the Neumann series

$$
g_{j}(\underline{r}, \underline{\omega})+\sum_{k=1}^{\infty} J_{k, j}(\underline{r}, \underline{\omega})
$$

converges to $\eta_{j}(\underline{r}, \underline{\omega})$ for all $(\underline{\underline{r}}, \underline{\omega})$ of $R$, where

$$
\begin{array}{r}
J_{k, j}(\underline{r}, \underline{\omega})=\int_{R} \ldots \int_{R} K\left(\underline{r}, \underline{\omega} ; \underline{r}_{k-1}, \underline{\omega}_{k-1}\right) \cdots K\left(\underline{r}_{1}, \underline{\omega}_{1} ; \underline{r}_{0}, \underline{\omega}_{0}\right) g_{j}\left(\underline{r}_{0}, \underline{\omega}_{0}\right) \\
\quad d \underline{r}_{0} \ldots d \underline{r}_{k-1} d \underline{\omega}_{0} \cdots d \underline{\omega}_{k-1} .
\end{array}
$$

The author knows of no way to establish these assumptions rigorously for the specific equations dealt with here. It is easy to show that, if a unique, non-negative, everywhere bounded solution of (5. 1) or (5.4) exists, then the corresponding Neumann series does converge to this solution. Thus, the assumptions may be partly justified on physical grounds.

Let $C=\left\{\left(\underline{r}_{0}, \underline{\omega}_{0} ; Q\right), \ldots,\left(\underline{r}_{n}(C)-1, \underline{\omega}_{n}(C)-1 ; Q\right),\left(\underline{r}_{n}(C), \underline{\omega}_{n}(C) ; P\right), \ldots\right\}$ be any point of the sample space $\Omega$ defined in Section IV; thus, by this notation, the Monte Carlo history $\mathrm{C}$ is terminated in the state $\left(\underline{r}_{n}(C), \underline{\omega}_{n}(C)\right)$ of $R$ after having made non-terminating collisions in the states $\left(\underline{r}_{0}, \underline{\omega}_{0}\right), \ldots$ $\left(\underline{r}_{n}(C)-1, \underline{\omega}_{n}(C)-1\right)$, in that order. Let $i$ denote any region of the geometry. Numbers $\xi_{i}(C)$ are associated with each history $C$. These numbers are defined by

$$
\xi_{i}(C)=\sum_{\ell=0}^{n(C)} g_{i}\left(\underline{r}_{\ell}, \underline{\omega}_{\ell}\right), i=0, \ldots, N-1,
$$

where $g_{i}$ is defined by $(5.2)$, with $x_{j}^{*}$ replaced by the characteristic function of all replicas of region $i$, and where $\mathrm{N}$ is the total number of regions of the geometry of the basic quarter-cell. Then, $\xi_{i}$ is a real-valued function on the sample space $\Omega$-which is measurable with respect to the analog measure $\mu$; i.e. $\xi_{i}$ is a real randorn variable on $\Omega$. In.theorem (8.68) of Ref 3 and in the discussion

†३ะé $\operatorname{Rel} 4$, p 206. 
preceding this theorem, it was shown that

$$
E\left[\xi_{i}\right] \equiv \int_{\Omega} \xi_{i} \mathrm{~d} \mu=\iint_{R} g_{i}(\underline{r}, \underline{\omega}) \psi_{N}(\underline{r}, \underline{\omega}) \mathrm{d} \underline{\mathrm{r}} \mathrm{d} \underline{\omega}
$$

under the assumptions made previously concerning the convergence of the Neumann series (5.5) and (5. 7). Equation (5.10) states that the random variable $\xi_{i}$ is an unbiased estimator of

$$
\iint_{R} g_{i}(\underline{r}, \underline{\omega}) \psi_{N}(\underline{r}, \underline{\omega}) d \underline{r} d \underline{\omega} \text {. }
$$

Notice that

$$
\xi_{i}(C)=\frac{\Sigma_{a, i}}{\Sigma_{t, i}} k_{i}(C)
$$

where $\Sigma_{a, i}, \Sigma_{t, i}$ are the macroscopic absorption and total cross sections, respectively, for region $i$, and where $k_{i}(C)$ is the number of collisions made by the history $C$ in all replicas of region $i$. Thus, Eq $(5.10)$ is equivalent to

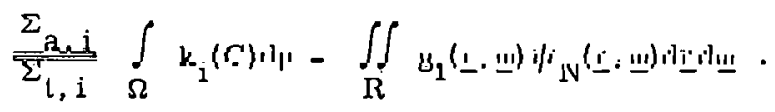

Now, a new random variable $\rho_{i}$ on $\Omega$ is defined by

$$
\rho_{i}(C)=\text { total distance }(\mathrm{cm}) \text { travelled by history } C \text { in all replicas of region } i \text {. }
$$

The constant $\Sigma_{t, i}$ is the expected number of collisions per centimeter in a region of total cross section $\Sigma_{t, i}$; however, by definition,

$$
E\left[k_{i}\right]=\int_{\Omega} k_{i}(C) d \mu=\begin{aligned}
& \text { expected total number of collisions } \\
& \text { in all replicas of region } i,
\end{aligned}
$$

while

$$
E\left[\rho_{i}\right]=\int_{\Omega} \rho_{i}(\mathrm{C}) \mathrm{d} \mu=\underset{\begin{array}{l}
\text { expected total distance } \\
\text { in all replicias of region } i .
\end{array}}{\mathrm{cm}} \text { travelled }
$$

Thus,

or

$$
\left.\begin{array}{rl}
z_{t, i} & =\frac{F\left[k_{i}\right]}{E\left[\rho_{i}\right]} \\
E\left[\rho_{i}\right] & =\frac{E\left[k_{i}\right]}{\Sigma} \sum_{t, i}
\end{array}\right\}
$$

From (5.12),

$$
\Sigma_{a, i} E\left[\rho_{i}\right]=\iint_{R} g_{i}(\underline{r}, \underline{\omega}) \psi_{N}(\underline{r}, \underline{\omega}) d \underline{r} \underline{d} \underline{\omega} .
$$

Now, random variables $\rho_{j}$ are defined by

$$
r_{j}=\sum^{\prime} \Sigma_{0,1} \rho_{1}
$$

where $\Sigma^{\prime}$ denotes the sum over all regions i forming composition $j$. Then,

$$
E\left[\rho_{j}\right]=\iint_{R} g_{j}(\underline{r}, \underline{\omega}) \psi_{N}(\underline{r}, \underline{\omega}) d \underline{r} d \underline{\omega},
$$

proving that $\rho_{j}$ is an unbiased estimator of $p_{j}$. The random variables $\rho_{j}$ are the ones used in TUT-T5. Observe that $\rho_{j}$ assigns to each chain $C$ the weight

$$
\rho_{j}(C)=\Sigma^{\prime} \Sigma_{a, i} d_{i}(C),
$$


where $d_{i}(C)$ is the distance travelled in all replicas of region $i$.

As mentioned in the introduction (Section I), it would be desirable to know the variance of $\rho_{j}$ ' since this would allow precise estimates of the expected statistical error as a function of the size of the sample. Unfortunately, the integrals which express these variances are not easily related to known parameters of the problem and, thus, estimates of the expected error are always obtained in practice by the code. These prove reliable for reasonably large samples and have served to show that the TUT-T5 estimators provide an efficient means of calculating capture probabilities.

\section{REFERENCES}

1. J. Spanier, H. Kuehn, and W. Guilinger, "TUT-T5-A Two-Dimensional Monte Carlo Calculation of Capture Probabilities for the IBM-704," WAPD-TM-125 (November 1959).

2. G. Goertzel and M. H. Kalos, "Monte Carlo Methods in Transport Problems," Progress in Nuclear Energy, Series I, Physics and Mathematics, Vol 2, D. H. Hughes, J. E. Sanders, and J. Horowitz, eds., Pergamon Press (1958).

3. J. Spanier, "Monte Carlo Methods and Their Application to Neutron Transport Problems," WA PD-195, (July, 1959).

4. B. Davison, Neutron Transport Theory, (Fair Lawn, New Jersey: Oxford University Press, 1957). 